\title{
APPROXIMATING INTEGRABLE SETS BY COMPACTS CONSTRUCTIVELY
}

\author{
BAS SPITTERS
}

\begin{abstract}
In locally compact spaces, (Borel-)measurable sets can be approximated by compact sets. Ulam extended this result to complete separable metric spaces. We give a constructive proof of Ulam's theorem. It is first proved intuitionistically and then, using a logical 'trick' due to Ishihara, a proof acceptable in Bishop-style mathematics is obtained. We feel this proof provides some insight into Ishihara's trick. Finally, we show how several intuitionistic measure theoretic theorems can be extended to regular integration spaces, that is integration spaces where integrable sets can be approximated by compacts. These results may help to understand Bishop's choice of definitions.
\end{abstract}

\section{INTRODUCTION}

When developing measure theory constructively, one often wants to know whether measurable sets can be approximated by compact sets. In fact, for Brouwer [Hey56, Bro75] this is part of the definition of a (Lebesgue-)measurable set. In Bishop's more abstract approach to integration theory one can prove that an integrable set, with respect to a positive measure on a locally compact space, can be approximated by a compact set, see Theorem 6.6.7 in [BB85].

In this article we will extend this result to integrals defined on complete separable metric spaces. We will work constructively, in the sense of Bishop, that is using intuitionistic logic. However, sometimes one of Brouwer's principles is used as an extra axiom. We will always make clear when we use such axioms. This treatment is similar to that of Bridges and Richman [BR87], who consider Brouwer's intuitionistic mathematics as an extension of Bishop's constructive mathematics. It should be noted that we do not work within a fixed formal system, but there have been various proposals for formalizing Bishop-style mathematics. See for instance [Bee85, TvD88].

This article is organized as follows. In section 2 we recall some standard result form the constructive literature. Section 3 gives an overview of the Bishop/Cheng integration theory. In section 4 we give a constructive proof of Ulam's theorem ${ }^{1}$. Finally, in section 5, we show how several intuitionistic measure theoretic theorems can be extended to regular integration spaces

\footnotetext{
${ }^{1}$ Billingsley [Bil68] refers to Ulam for this result. Unfortunately, I have been unable to find a concrete reference to Ulam's work.
} 


\section{Preliminaries}

We will now recall some results and fix some notations, most of them are standard in the literature on constructivism, see for instance [Bee85, TvD88].

Definition 2.1. Let $\operatorname{Seq}(\mathbf{N})$ be the set of finite sequences of natural numbers. The concatenation of two finite sequences is denoted by $*$ and we write $a * n$ for $a *\{n\}$, when $a \in \operatorname{Seq}(\mathbf{N})$ and $n \in \mathbf{N}$. Define the map ${ }^{-}: \mathbf{N}^{\mathbf{N}} \times \mathbf{N} \rightarrow \operatorname{Seq}(\mathbf{N})$ such that for all $\alpha \in \mathbf{N}^{\mathbf{N}}$ and $n \in \mathbf{N}, \bar{\alpha} n$ is the initial segment of $\alpha$ which has length $n$. When $a$ is an initial segment of a finite sequence $b$ we write $a \subset b$. When $a$ is an initial segment of an infinite sequence $\alpha$, we write $\alpha \in a$.

Let $d$ be the metric on $\mathbf{N}^{\mathbf{N}}$ defined by

$$
d(\alpha, \beta):=\inf \left\{2^{-n}: \bar{\alpha} n=\bar{\beta} n\right\} .
$$

We will sometimes implicitly consider $\mathbf{N}^{\mathbf{N}}$ as the metric space $\left(\mathbf{N}^{\mathbf{N}}, d\right)$ without mentioning it.

Definition 2.2. A spread $S$ is a decidable subtree of $\operatorname{Seq}(\mathbf{N})$ such that

(1) when there exists $n \in \mathbf{N}$ such that $a * n \in S$, then $a \in S$ and

(2) if $a \in S$, then $a * n \in S$, for some $n$.

A spread $S$ can also be seen closed subset of $\mathbf{N}^{\mathbf{N}}$ by considering the set $\{\alpha \in$ $\left.\mathbf{N}^{\mathbf{N}}: \forall n[\bar{\alpha} n \in S]\right\}$. In this case we also write $\alpha \in S$, for $\alpha \in \mathbf{N}^{\mathbf{N}}$. A fan is a spread $F$ which is finitely branching. Let $S$ be a spread. A bar $B$ for $S$ is a set of finite sequences of natural numbers such that for all infinite sequences $\sigma \in S$ there is $b \in B$ such that $\sigma \in b$. A subbar of a bar $B$ is a subset of $B$ that is a bar.

The fan theorem and the continuity principle are two of the most characteristic principles of intuitionistic mathematics. Brouwer considered them to be theorems. Bishop and Bridges [BB85, p.76] seem to believe that these principles hold, but they do not consider them to be mathematical theorems. They avoid using these principles by adapting their definitions. The most well-known example of this is their definition of continuity. They define a function on a locally compact space to be continuous precisely when it is uniformly continuous on compacts. For Bishop a subset of a metric space is compact, if it is totally bounded and complete. This is also the definition we will use.

We think that the intuitionists have made it clear that it is worthwhile to find out what the consequences of these statements are, so we will treat these principles as axioms. We will always make it clear when we use these axioms.

Axiom 2.3. The continuity principle $(\mathrm{CP})$ : Let $S$ be a spread and let $A \subset S \times \mathbf{N}$. If for all $\alpha \in S$ there is an $n \in N$ such that $A(\alpha, n)$, then for each $\alpha \in S$, there are $n, m \in \mathbf{N}$ such that if $\beta \in S$ and $\bar{\beta} m=\bar{\alpha} m$, then $A(\beta, n)$.

The axiom $\mathrm{CP}$ is also know as WC-N. This principle also holds when interpreted in Markov's recursive mathematics. 
Axiom 2.4. The fan theorem(FAN): Let $F$ be a fan and $B$ a decidable bar for $F$, then there is a finite subbar $B^{\prime} \subset B$ which is also a bar for $F$.

Note that the fan theorem also holds when read classically.

Axiom 2.5. The extended fan theorem $\left(\mathrm{FAN}_{\mathrm{ext}}\right)$ : Let $F$ be a fan and let $A \subset$ $F \times \mathbf{N}$. If for all $\alpha \in F$ there is $n \in \mathbf{N}$ such that $A(\alpha, n)$, then there is $N \in \mathbf{N}$ such that for all $\alpha \in F$ there is an $n \leq N$ such that $A(\alpha, n)$.

The following theorems are useful for later reference. They can be found for instance as Corollary 5.2.4, Corollary 5.3.7 and Theorem 5.3.6 in [BR87].

Theorem 2.6. [CP] Every function on a complete separable metric space is (pointwise) continuous.

Theorem 2.7. [FAN] Let $h$ be a continuous function on $[0,1]$ such that for all $x \in[0,1], h(x)>0$. Then there is $\varepsilon>0$ such that $h(x)>\varepsilon$, for all $x \in[0,1]$.

Theorem 2.8. [FAN] Every pointwise continuous function on a compact space is uniformly continuous.

2.1. Ishihara's trick. We will now present what we feel is the essence of what has come to be known as Ishihara's trick, see for instance in [Ish91, BvDI03]. The trick provides a way to find a proof which is acceptable in Bishop-style mathematics for certain statements for which we have both an intuitionistic and a classical proof. This technique rests on two observations. First, many theorems in classical analysis can be proved in Bishop-style mathematics assuming only LPO, instead of the full form of the law of excluded middle. Here LPO denotes the 'limited principle of omniscience',

$$
\forall \alpha \in \mathbf{N}^{\mathbf{N}}[\exists n[\alpha(n) \neq 0] \vee \forall n[\alpha(n)=0]] .
$$

Second, given a discontinuous function on a complete metric space we can prove LPO. In view of theorem 2.6 no such function exist in intuitionistic mathematics.

A precise statement of Ishihara's trick is the following.

Theorem 2.9. Let $(X, d)$ be a complete metric space. Let $f$ be a strongly extensional map from $X$ to a metric space $(Y, \rho)$, and let $\left(x_{n}\right)_{n \in \mathbf{N}}$ be a sequence in $X$ converging to a point $x \in X$. Then for all $\epsilon>0$, either $L P O$ or $\rho\left(f\left(x_{n}\right), f(x)\right) \leq \epsilon$ for all sufficiently large $n$.

A map $f$ between a metric space $X$ and a metric space $Y$ is called strongly extensional when $f(x) \neq f(y)$, whenever $x, y$ in $X$ and $x \neq y$. Here we use Bishop's notation where $a \neq b$ denotes that $a$ is apart from $b$, that is the distance between $a$ and $b$ is positive. Every continuous function is strongly extensional.

It should be noted that the theorem is trivial in classical, intuitionistic and recursive mathematics. Indeed, in classical mathematics LPO holds. In intuitionistic and recursive mathematics every function on a complete separable metric 
space is pointwise continuous, so one can consider a complete separable subspace of $X$ containing the sequence $\left(x_{n}\right)_{n \in \mathbf{N}}$.

We now outline a proof of Ishihara's trick in three lemmas. Let $(X, d)$ be a complete metric space. Let $f$ be a strongly extensional map from $X$ to a metric space $(Y, \rho)$, let $\epsilon>0$ and let $\left(x_{n}\right)_{n \in \mathbf{N}}$ be a sequence in $X$ converging to a point $x \in X$.

Lemma 2.10. Either for all $n,\left|f(x)-f\left(x_{n}\right)\right|<2 \varepsilon$ or there exists an $n$ such that $\left|f(x)-f\left(x_{n}\right)\right|>\varepsilon$.

Proof. Define an increasing binary sequence such that

$$
\begin{aligned}
& \lambda(n)=0 \Rightarrow\left|f(x)-f\left(x_{n}\right)\right|<2 \varepsilon ; \\
& \lambda(n)=1 \Rightarrow\left|f(x)-f\left(x_{m}\right)\right|>\epsilon, \text { for some } m \leq n .
\end{aligned}
$$

Define $y_{n}=x_{n}$ when $\lambda(n)=0$ and $y_{n}=x_{n_{0}}$ when $n_{0} \leq n$ is the first $m$ such that $\lambda(m)=1$. The sequence $y_{n}$ converges to a limit $y$. If $|f(y)-f(x)|<\varepsilon$, then for all $n,\left|f(x)-f\left(x_{n}\right)\right|<2 \varepsilon$. If on the other hand $|f(y)-f(x)|>\epsilon / 2$, then there exists an $n$ such that $\left|f(x)-f\left(x_{n}\right)\right|>\varepsilon$.

Lemma. Either for sufficiently large $n,\left|f(x)-f\left(x_{n}\right)\right|<2 \varepsilon$ or there are infinitely $n$ such that $\left|f(x)-f\left(x_{n}\right)\right|>\varepsilon$.

Proof. Using the previous lemma we define an increasing binary sequence such that

$$
\begin{aligned}
& \lambda(n)=0 \Rightarrow \text { there exists } m>n,\left|f(x)-f\left(x_{m}\right)\right|>\varepsilon \\
& \lambda(n)=1 \Rightarrow \text { for all } m>n,\left|f(x)-f\left(x_{m}\right)\right|<2 \epsilon .
\end{aligned}
$$

Define $y_{n}=x_{n}$ when $\lambda(n)=0$ and $y_{n}=x_{n_{0}}$ when $n_{0} \leq n$ is the first $m$ such that $\lambda(m)=1$. The sequence $y_{n}$ converges to a limit $y$. If $|f(y)-f(x)|<\varepsilon$, then here are infinitely many $n$ such that $\left|f(x)-f\left(x_{n}\right)\right|>\varepsilon$. If $|f(x)-f(y)|>\epsilon / 2$ then for sufficiently large $n,\left|f(x)-f\left(x_{n}\right)\right|<2 \varepsilon$.

Corollary. Either for sufficiently large $n,\left|f(x)-f\left(x_{n}\right)\right|<2 \varepsilon$ or LPO.

\section{INTEGRATION THEORY}

In this section we recall some results from Bishop and Cheng's constructive version of the Daniell's integration theory [BC72, BB85].

A triple $(X, L, I)$ is an integration space if $X$ is an inhabited set with an apartness relation $\neq, L$ a set of strongly extensional partial functions, and $I$ is a mapping from $L$ into $\mathbf{R}$, called the integral, such that the following properties hold.

(1) If $f, g \in L$ and $\alpha, \beta \in \mathbf{R}$, then $\alpha f+\beta g,|f|$ and $f \wedge 1$ belong to $L$, and $I(\alpha f+\beta g)=\alpha I(f)+\beta I(g)$. 
(2) If $f \in L$ and $\left(f_{n}\right)_{n \in \mathbf{N}}$ is a sequence of nonnegative functions in $L$ such that $\sum_{n=1}^{\infty} I\left(f_{n}\right)$ converges and $\sum_{n=1}^{\infty} I\left(f_{n}\right)<I(f)$, then there exists $x$ in $X$ such that $\sum_{n=1}^{\infty} f_{n}(x)$ converges and $\sum_{n=1}^{\infty} f_{n}(x)<f(x)$.

(3) There exists a function $p$ in $L$ with $I(p)=1$.

(4) For each $f$ in $L, \lim _{n \rightarrow \infty} I(f \wedge n)=I(f)$ and $\lim _{n \rightarrow \infty} I\left(|f| \wedge n^{-1}\right)=0$.

When $R$ denotes the Riemann integral, then $([0,1], C[0,1], R)$ is an example of an integration space. More generally, let $X$ be a locally compact space and $I$ any nonzero positive linear functional on the space of test functions $C(X)$; then $(X, C(X), I)$ is an integration space. A linear functional $I$ on $C(X)$ is called positive if $I(f) \geq 0$ whenever $f \geq 0$. Such a positive linear functional is also called a positive measure.

There is a general completion construction for integration spaces, which allows us to carry out all the usual measure theoretic constructions. The elements of the completion are, almost everywhere defined, partial functions. When this completion is applied to the Riemann integral one obtains the Lebesgue integral.

Intuitively, an integrable set is identified with its characteristic function $\chi_{A}$. To make this work nicely, one has to consider what is called a complemented set, that is a pair $\left(A^{1}, A^{0}\right)$ such that for all $a_{1} \in A^{1}$ and $a_{0} \in A^{0}, a_{1} \neq a_{0}$. When $\mu$ is an integral and $A$ a $\mu$-integrable (complemented) set we will usually write $\mu(A)$ instead of $\mu\left(\chi_{A}\right)$. Similarly, we will sometimes use the notation $\int f d \mu$ instead $\mu(f)$.

The following theorem is a fundamental result in integration theory. Let $f$ be an integrable function and let $\alpha>0$. Define the complemented sets

$$
\begin{aligned}
& {[f \geq \alpha]:=(\{x: f(x) \geq \alpha\},\{x: f(x)<\alpha\}) \text { and }} \\
& {[f>\alpha]:=(\{x: f(x)>\alpha\},\{x: f(x) \leq \alpha\}) .}
\end{aligned}
$$

In general, we will not be able to compute the measure of the set $[f>\alpha]$ for all $\alpha$. However, the next theorem states that $I([f>\alpha])$ can be computed for many $\alpha$.

Let $T$ be a countable subset of a metric space $X$ and write $X \sim T$ for the set $\{x \in X ; \forall t \in T x \neq t\}$. When a predicate $P$ holds for all $x \in X \sim T$, we will say that $P$ holds for all but countably many $x$.

Theorem 3.1. Let $I$ be an integral and let $f$ be an integrable function; then for all but countably many $\alpha>0$, the sets $[f \geq \alpha]$ and $[f>\alpha]$ are integrable and have the same measure. Moreover, for each admissible $\alpha>0$ and each $\varepsilon>0$, there is $\delta>0$ with

$$
\left|I[f \geq \alpha]-I\left[f \geq \alpha^{\prime}\right]\right|<\varepsilon,
$$

whenever $\alpha^{\prime}>0$ is admissible and $\left|\alpha-\alpha^{\prime}\right|<\delta$.

In the context of the previous theorem, $\alpha$ is called admissible for $f$, if $\alpha$ is admissible for the property 'the sets $[f \geq \alpha]$ and $[f>\alpha]$ are integrable and have the same measure'. This theorem can be read as a constructive way of stating 
that the function $\alpha \mapsto I[f \geq \alpha]$ being non-decreasing, is continuous in all but countably many points.

It is important to define measurable functions together with an integral. So the following definition may look different from the ones the reader is used to.

An integration space $X$ is finite when $X$ is integrable and (therefore) $\mu(X)<$ $\infty$. We will not recall the general definition of a full set, but in the special case when the integration space $X$ is finite, a set $A$ is full when $\mu(A)=\mu(X)$.

Definition 3.2. A function defined on a full set is measurable if for each integrable set $A$ and each $\varepsilon>0$, there exist an integrable set $B \subset A$ and an integrable function $g$ such that $\mu(A-B)<\varepsilon$ and $|f-g|<\varepsilon$ on $B$.

\section{Regular MeAsures AND Ulam's theOREM}

In this section we study regular measures and prove Ulam's theorem. Regularity is very useful when combined with intuitionistic axioms, as we will show in section 5 .

We want to find a good substitute for the classical notion of a Borel measure on a complete separable metric space. Classically, a Borel measure is a measure such that all open sets and hence all Borel sets are measurable. Constructively, even for Lebesgue measure on $[0,1]$ not all open sets are measurable. For measures on locally compact spaces a good substitute is to demand that all test functions, and hence all uniformly continuous functions are measurable. We define a Borel measure as a measure on a separable metric space such that all uniformly continuous functions are measurable.

There is an example in recursive mathematics of a pointwise continuous, but not uniformly continuous, function on $[0,1]$ that is not Lebesgue measurable, see for instance [BD91, Cor. 1, p.272].

Recall that a total function $f$ on a metric space $X$ is called Lipschitz if there is a constant $L>0$ such that for all $x, y \in X,|f(x)-f(y)| \leq L \rho(x, y)$. Define for all $x \in X$, the Lipschitz function $\rho_{x}$ by $\rho_{x}(y):=\rho(x, y)$. Then for all $x \in X$ and $\varepsilon>0, B(x, \varepsilon)=\left[\rho_{x}<\varepsilon\right]$. Using classical logic, it follows that all basic open sets, and hence all open sets, are measurable. Consequently, the present definition of Borel measure is equivalent to the usual one in classical mathematics.

Definition 4.1. Let $X$ be a metric space. A measure $\mu$ on $X$ is regular if for every measurable set $A \subset X$ and $\varepsilon>0$ there is a compact integrable set $C \subset A$ such that $\mu(A-C)<\varepsilon$. A finite measure $\mu$ on $X$ is tight if for each $\varepsilon>0$ there is a compact integrable set $C \subset X$ such that $\mu(X-C)<\varepsilon$.

What we call 'regular' is called 'inner regular for compacts' by some authors.

Positive measures on locally compact sets are regular [BB85, Thm. 6.6.7]. Theorem 4.3 states that many more measures are regular.

Lemma 4.2. A tight finite Borel measure is regular. 
Proof. Let $\mu$ be a tight Borel measure on a metric space $X$ and $\varepsilon>0$. We may assume that $\mu(X)=1$. Choose a compact integrable set $A \subset X$ such that $\mu(A)>\mu(X)-\varepsilon / 2$ and apply Theorem 6.6.7 [BB85, p.257] to the restriction of $\mu$ to $A$.

Theorem 4.3. [Ulam] Let $X$ be a complete separable metric space and let $\mu$ be a finite Borel measure on $X$. Then $\mu$ is regular.

We will first give a classical proof of this theorem due to Ulam (see [Bil68, p.10]). We then give an intuitionistic proof using $\mathrm{CP}$, and finally we will use Ishihara's trick to remove this hypothesis and obtain a proof which is acceptable in Bishop-style mathematics.

We assume that $\mu(X)=1$.

Classical proof. Let $Q=\left\{q_{1}, q_{2}, \ldots\right\}$ be a countable dense set in $X$. Let $\varepsilon>0$ and $m \in \mathbf{N}$. Because $X=\bigcup_{n=1}^{\infty} B\left(q_{n}, 1 / m\right)$, there is $N_{m}$ such that

$$
\mu\left(\bigcup_{n=1}^{N_{m}} B\left(q_{n}, 1 / m\right)\right)>1-\varepsilon 2^{-m} .
$$

Define $C:=\bigcap_{m} \bigcup_{n=1}^{N_{m}} \overline{B\left(q_{n}, 1 / m\right)}$. Then $C$ is compact and $\mu(C) \geq 1-\varepsilon$.

There are two main problems when we try to interpret the previous proof constructively. First, we can not conclude in general that if $\left(X_{n}\right)_{n \in \mathbf{N}}$ is a sequence of integrable sets and $X=\bigcup_{n=1}^{\infty} X_{n}$, then there exists a natural number $N$ such that $\mu\left(\bigcup_{n=1}^{N} X_{n}\right)>1 / 2$. Secondly, it is not clear constructively that a set like the set $C$ defined is totally bounded, one has to choose an apropriate subset of $\left\{q_{1}, \ldots, q_{N_{m}}\right\}$ as an $1 / m$-net, depending on the choices made for $k<m$.

We elaborate on the first problem. In recursive mathematics there is a sequence $\left(I_{n}\right)_{n \in \mathbf{N}}$ of intervals such that $[0,1]=\bigcup_{n=1}^{\infty} I_{n}$ but for all $N \in \mathbf{N}, \mu\left(\bigcup_{n=1}^{N} I_{n}\right)<$ $1 / 2$, see for instance [BD91]. Here $\mu$ denotes Lebesgue measure, which is regular. In intuitionistic mathematics, when $\left(X_{n}\right)_{n \in \mathbf{N}}$ is a sequence of integrable sets and $X=\bigcup_{n=1}^{\infty} X_{n}$, then there exists a natural number $N$ such that $\mu\left(\bigcup_{n=1}^{N} X_{n}\right)>1 / 2$, for any measure $\mu$ which is regular (see Theorem 5.5). However, regularity is exactly what we are trying to prove!

With these problems in mind it may seem quite surprising that it is possible to proof Ulam's theorem constructively. In fact, the only way we know to do this is by first given an intuitionistic proof and then use Ishihara's trick to transform this into a constructive proof. We will now proceed towards an intuitionistic proof of Ulam's theorem. Let $\overline{\mathbf{N}}$ be the completion of $\mathbf{N}$ with respect to the metric $d(n, m):=|1 /(n+1)-1 /(m+1)|$ and let $\infty$ be the point at infinity. Remark that $\overline{\mathbf{N}}$ is a complete separable metric space.

Proof using CP. It follows from Lemma 4.2 that we only need to prove that $\mu$ is tight. To do so we first prove a lemma which contains the key argument of the proof. 
Lemma. Let $X$ be a complete separable metric space, $D$ a finite subset of $X$ and $\mu$ be a finite Borel measure on $X$. Then for each $\varepsilon>0$, there is a closed separable $\mu$-measurable subset $A$ of $X$ such that there is a finite $\varepsilon$-net for $A$ which contains $D$ and $\mu(X)-\mu(A)<\varepsilon$.

Proof of the lemma. Write $D=\left\{q_{1}, \ldots, q_{d}\right\}$ and choose a countable dense set $Q=\left\{q_{1}, q_{2}, \ldots\right\}$ in $X$. Let $T:=\{\rho(x, y): x, y \in Q\}$, then $T$ is countable. Note that for all $s \in X \sim T$ and $x, y \in Q$, we can decide whether $x \in B(y, s)$ or not. Define for all $N \in \mathbf{N}$,

$$
h_{N}(x):=\min _{n \leq N} \rho\left(x, q_{n}\right) \wedge 1 .
$$

For all $N \in \mathbf{N}$ and all $\alpha \in(0,1] \sim T$ which are admissible for $h_{N}$,

$$
\left[h_{N}<\alpha\right]=\left\{x: \min _{n \leq N} \rho\left(x, q_{n}\right)<\alpha\right\}=\bigcup_{n=1}^{N} B\left(q_{n}, \alpha\right) .
$$

Since $\alpha$ is admissible for $h_{N}, \mu\left[h_{N}<\alpha\right]=\mu\left[h_{N} \leq \alpha\right]$ and, because $h_{N}$ is continuous,

$$
\left[h_{N} \leq \alpha\right] \supset \overline{\bigcup_{n=1}^{N} B\left(q_{n}, \alpha\right)} .
$$

For all $n \in \mathbf{N}, h_{n}$ is Lipschitz with constant 1 . Because $Q$ is dense the sequence $\left(h_{n}\right)_{n \in \mathbf{N}}$ converges pointwise to 0 . So the map $n \mapsto h_{n}$ can be extended from $\mathbf{N}$ to $\overline{\mathbf{N}}$ such that $h_{\infty}=0$. Then $h_{\beta}$ is Lipschitz with constant 1 , for all $\beta \in \overline{\mathbf{N}}$.

The function $f: \overline{\mathbf{N}} \rightarrow \mathbf{R}$ defined by $f(\beta):=\mu\left(h_{\beta}\right)$ is continuous, by CP. Because $f(\infty)=0$, there is an $N$ such that $\mu\left(h_{N}\right)<\varepsilon$. For all positive integrable $g$ and every $\alpha>0$ which is admissible for $g, \alpha \mu[g \geq \alpha] \leq \int g$. So for fixed $\alpha>0$, $\mu\left[h_{N} \geq \alpha\right] \rightarrow 0$.

Choose $N \geq d$ and $\alpha \in(0, \varepsilon / 2] \sim T$ such that $\alpha$ is admissible for $h_{N}$ and $\mu\left[h_{N}<\alpha\right]>\mu(X)-\varepsilon$. Define $A:=\overline{\left[h_{N}<\alpha\right]}$. We claim that $A$ satisfies the required properties. We only show that $A$ is separable. Indeed, $A$ is separable, because for all $q \in Q$, we can decide whether $q \in \bigcup_{n=1}^{N} B\left(q_{n}, \alpha\right)$ or not. So $\bigcup_{n=1}^{N} B\left(q_{n}, \alpha\right)$ is separable and hence $A$ is separable.

We now complete the intuitionistic proof of Ulam's theorem by appling the Lemma to obtain better and better approximations of the compact set.

Let $\varepsilon>0$. Let $A_{1}$ be a closed separable $\mu$-measurable subset of $X$ and a finite $\varepsilon$-net $D_{1}$ for $A_{1}$ such that $\mu(X)-\mu\left(A_{1}\right)<2^{-1} \varepsilon$. Suppose that there are closed separable $\mu$-measurable subsets $A_{1}, \ldots, A_{n} \subset X$ such that for all $i<n, A_{i+1} \subset A_{i}$ and $\mu\left(A_{i}\right)-\mu\left(A_{i+1}\right)<2^{-i} \varepsilon$. Moreover, suppose that finite sets $D_{1}, \ldots, D_{n} \subset X$ have been defined such that for all $i<n, D_{i} \subset D_{i+1}$ and for all $i \leq n, D_{i}$ is an $2^{-i} \varepsilon$-net for $A_{i}$. Remark that $A_{n}$ is a complete separable metric space. So we can apply the lemma to the space $A_{n}$, the set $D_{n}$ and the restriction of $\mu$ 
to $A_{n}$ to obtain a closed separable $\mu$-measurable set $A_{n+1} \subset A_{n}$ and a finite set $D_{n+1} \subset A_{n+1}$ such that $\mu\left(A_{n}\right)-\mu\left(A_{n+1}\right)<2^{-n} \varepsilon, D_{n} \subset D_{n+1}$ and $D_{n+1}$ is an $2^{-(n+1)} \varepsilon$-net for $A_{n+1}$. It follows that $A:=\bigcap_{i=1}^{\infty} A_{i}$ is a compact set such that $\mu(X)-\mu(A)<\varepsilon$.

To remove the assumption of CP from the proof above we can use Ishihara's trick. The function $f$ we defined above is a strongly extensional map on the complete space $\overline{\mathbf{N}}$, by [BB85, Lemma 6.1.3]. Let $\varepsilon>0$. By Ishihara's trick, either for all sufficiently large $n, f(n)<\varepsilon$ or LPO. We assume the latter. In this case the descending sequence $f(n)=\mu\left(h_{n}\right)$ of non-negative real numbers converges. So the sequence $\left(h_{n}\right)_{n \in \mathbf{N}}$ is Cauchy in $L_{1}$, and as it converges pointwise to 0 , it converges in $L_{1}$ to the constant function with value 0 . Hence $\lim _{n \rightarrow \infty} \mu\left(h_{n}\right)=0$. We conclude that in both cases we can compute an $n \in \mathbf{N}$ such that $f(n)<\varepsilon$ and continue as in the proof above.

\section{InTUITIONISTIC THEOREMS}

Every compact set can be represented as the image of a fan (see for instance [TvD88, p.363]). This fact can be used to obtain the following four striking results. Similar results were first proved by van Rootselaar [vR54, Hey56] for Lebesgue measure on $[0,1]$. The first theorem was proved for positive measures on locally compact spaces by Bridges and Demuth in [BD91]. They also used intuitionistic axioms.

Theorem 5.1. [FAN,CP] Let $\mu$ be a regular measure on a metric space $X$. Every $\mu$-a.e. defined function is measurable.

Proof. Let $\varepsilon>0$. Let $f$ be an a.e. defined function and let $A$ be an integrable set. Choose a compact integrable set $C \subset A \cap \operatorname{Dom} f$ such that $\mu(A-C)<\varepsilon$. By FAN and CP, $f$ is uniformly continuous on $C$. Construct by the Tietze extension theorem [BB85, Thm. 4.6.16] a bounded uniformly continuous extension $g$ of $\left.f\right|_{C}$ to $X$. Then $g \chi_{C}$ is integrable and $f=g$ on $C$. So $f$ is measurable.

A simple, but interesting consequence is the following. If $f$ is measurable on a regular measure space and $g$ is a measurable function on $\mathbf{R}$, such that $f(x) \in$ Dom $g$ for almost all $x$, then $g \circ f$ is measurable. In particular, if $f>0$, then one may choose $g(x):=x^{-1}$ on $(0, \infty)$. This should be compared with [BB85, Cor. 6.7.10], where stronger assumptions are necessary to prove that $1 / f$ is measurable.

The hypothesis that $f(x) \in$ Dom $g$ for almost all $x$ is necessary, because, in order to be measurable, the function $g \circ f$ needs to be defined on a full set.

Definition 5.2. Let $\left(f_{n}\right)_{n \in \mathbf{N}}$ be a sequence of measurable functions, and $f$ a function defined on a full set. The sequence $\left(f_{n}\right)_{n \in \mathbf{N}}$ converges to $f$ almost uniformly if to each integrable $A$ and $\varepsilon>0$, there is an integrable $B \subset A$ with $\mu(A-B)<\varepsilon$ and the sequence $\left(f_{n}\right)_{n \in \mathbf{N}}$ converges uniformly on $B$. 
Theorem 5.3. [Egoroff] Let $\mu$ be a regular finite measure on a metric space. If a sequence $\left(f_{n}\right)_{n \in \mathbf{N}}$ of measurable functions converges on a full set to $f$, then the sequence $\left(f_{n}\right)_{n \in \mathbf{N}}$ converges almost uniformly.

Proof. [FAN $\mathrm{Fxt}_{\text {ext }}$ The sequence $\left(f_{n}\right)_{n \in \mathbf{N}}$ converges to $f$ on a large compact set. By the extended fan theorem it converges uniformly on this set.

Bishop and Bridges [BB85, Thm. 6.8.16] proved the previous theorem without the fan theorem, replacing convergence on a full set by the following strong definition of convergence a.e.

Definition 5.4. Let $\left(f_{n}\right)_{n \in \mathbf{N}}$ be a sequence of measurable functions, and $f$ a function defined on a full set. The sequence $\left(f_{n}\right)_{n \in \mathbf{N}}$ converges to $f$ almost everywhere if to each integrable $A$ and $\varepsilon>0$, there is $N \in \mathbf{N}$ and an integrable $B \subset A$ with $\mu(A-B)<\varepsilon$ and $\left|f-f_{n}\right|<\varepsilon$ on $B$ for all $n \geq N$.

The following result motivates this definition of convergence a.e. Again we see that Bishop avoids the use of intuitionistic axioms by modifying the more familiar definitions.

Lemma 5.5. $\left[\mathrm{FAN}_{\text {ext }}\right]$ Let $\mu$ be a regular measure. If a sequence $\left(f_{n}\right)_{n \in \mathbf{N}}$ of measurable functions converges pointwise on a full set, then the sequence $\left(f_{n}\right)_{n \in \mathbf{N}}$ converges almost everywhere.

Proof. Let $A$ be integrable and let $\epsilon>0$ compact set $C \subset A$, such that $\mu(A-C)<$ $\varepsilon$. By the extended fan theorem the sequence $\left(f_{n}\right)_{n \in \mathbf{N}}$ converges uniformly on this set.

Note that the fan theorem is crucial here. There is an example in recursive mathematics [BD91, Cor. 1, p.272] where $f$ is the pointwise limit of uniformly continuous functions on $[0,1], f$ is pointwise continuous and bounded, but not Lebesgue integrable.

Note that Lemma 5.5 can also be proved classically.

Theorem 5.6. [Lusin] Let $\varepsilon>0, \mu$ be a regular measure on a metric space and let $A$ be an integrable set. If $f$ is measurable then there exist an integrable $B \subset A$ such that $\mu(A-B)<\varepsilon$ and $f$ is uniformly continuous on $B$.

Proof. [FAN,CP] Choose a large compact set $C \subset A$ on which $f$ is defined. By FAN and CP $f$ is uniformly continuous on $C$.

A similar result can also be obtained in Bishop-style mathematics, but then the proof is somewhat longer:

Theorem 5.7. [Lusin] Let $\varepsilon>0, \mu$ be a regular positive measure on a locally compact space and let $A$ be an integrable set. If $f$ is measurable then there exist an integrable $B \subset A$ such that $\mu(A-B)<\varepsilon$ and $f$ is uniformly continuous on $B$. 
Proof. First assume that $f$ is a characteristic function of an integrable set $B=$ $\left(B^{1}, B^{0}\right)$. Take compact sets $B_{1} \subset B^{1}$ and $B_{0} \subset B^{0}$ such that $\mu\left(B^{i}-B_{i}\right)<\varepsilon / 2$ for all $i \in\{0,1\}$ and the distance between $B_{0}$ and $B_{1}$ is strictly positive, see Theorem 6.6.7 in [BB85] for this last fact. The function $f$ is constant on each $B_{i}$ and hence uniformly continuous on $B_{0} \cup B_{1}$. When $f$ is a simple function, we can construct in a similar way a large integrable set on which $f$ is uniformly continuous. Now let $f$ be the limit of a sequence $\left(f_{k}\right)_{k \in \mathbf{N}}$ of simple functions. There is a sequence $\left(C_{k}\right)_{k \in \mathbf{N}}$ of measurable sets such that for all $k \in \mathbf{N}, \mu(A-$ $\left.C_{k}\right)<\varepsilon 2^{-k-1}$ and $f_{k}$ is uniformly continuous on $C_{k}$. By applying Theorem 5.3 to this sequence of functions we find an integrable set $C_{0}$ such that $\mu\left(A-C_{0}\right)<\varepsilon / 4$ and the sequence $\left(f_{n}\right)_{n \in \mathbf{N}}$ converges uniformly on $C_{0}$. Now there is a measurable set $C \subset C_{0} \cap \bigcap_{k=1}^{\infty} C_{k}$, such that $\mu(A-C)<\varepsilon$. It follows that $f$ is uniformly continuous on $C$.

\section{Conclusions}

We have shown how to constructively approximate integrable sets by compact integrable sets. The proof uses an interesting logical detour, the theorem was first proved intuitionistically and only then these intuitionistic assumptions where removed to obtain a result acceptable in Bishop's constructive mathematics.

Finally, it is also possible to develop integration theory algebraically, see for instance [Spi04]. This seems to be more appropriate for the applications to functional analysis. However, the algebraic approach does not include a notion of convergence almost everywhere.

Acknowledgement. Some of the results in this article can also be found in the author's PhD thesis [Spi02]. The author would like to thank Wim Veldman for his advise during the $\mathrm{PhD}$ project and for remarks on an earlier version of this paper.

\section{REFERENCES}

[BB85] Errett Bishop and Douglas Bridges, Constructive analysis, Grundlehren der Mathematischen Wissenschaften, vol. 279, Springer-Verlag, 1985.

[BC72] Errett Bishop and Henry Cheng, Constructive measure theory, American Mathematical Society, Providence, R.I., 1972, Memoirs of the American Mathematical Society, No. 116.

[BD91] Douglas Bridges and Osvald Demuth, On the Lebesgue measurability of continuous functions in constructive analysis., Bulletin of the American Mathematical Society, New Series 24 (1991), no. 2, 259-276.

[Bee85] Michael J. Beeson, Foundations of constructive mathematics, Springer-Verlag, Berlin, Heidelberg, New York, 1985.

[Bil68] Patrick Billingsley, Convergence of probability measures, John Wiley and sons, New York, 1968.

[BR87] Douglas Bridges and Fred Richman, Varieties in constructive mathematics, London Mathematical Society Lecture Notes Series, no. 97, Cambridge University Press, 1987 (English). 
[Bro75] L.E.J. Brouwer, Collected works, North-Holland, 1975.

[BvDI03] D. Bridges, D. van Dalen, and H. Ishihara, Ishihara's proof technique in constructive analysis, Proc. Koninklijke Nederlandse Akad. Wetenschappen (Indag. Math.) N.S. 4 (2003), no. 2, 2749-2752.

[Hey56] Heyting, A., Intuitionism. An introduction., Studies in Logic and the Foundations of Mathematics, North-Holland Publishing Company, Amsterdam, 1956 (English).

[Ish91] Hajime Ishihara, Continuity and nondiscontinuity in constructive mathematics., Journal of Symbolic Logic 56 (1991), no. 4, 1349-1354.

[Spi02] Bas Spitters, Constructive and intuitionistic integration theory and functional analysis, Ph.D. thesis, University of Nijmegen, 2002.

[Spi04] — Constructive algebraic integration theory, Proceedings of the Second Workshop on Formal Topology, special issue of Annals of Pure and Applied Logic (Giovanni Sambin Bernhard Banaschewski, Thierry Coquand, ed.), 2004.

[TvD88] A.S. Troelstra and D. van Dalen, Constructivism in mathematics. An introduction. Volume II., Studies in Logic and the Foundations of Mathematics, no. 123, NorthHolland, 1988.

[vR54] B. van Rootselaar, Generalization of the brouwer integral, Ph.D. thesis, Universiteit van Amsterdam, Amsterdam, 1954. 